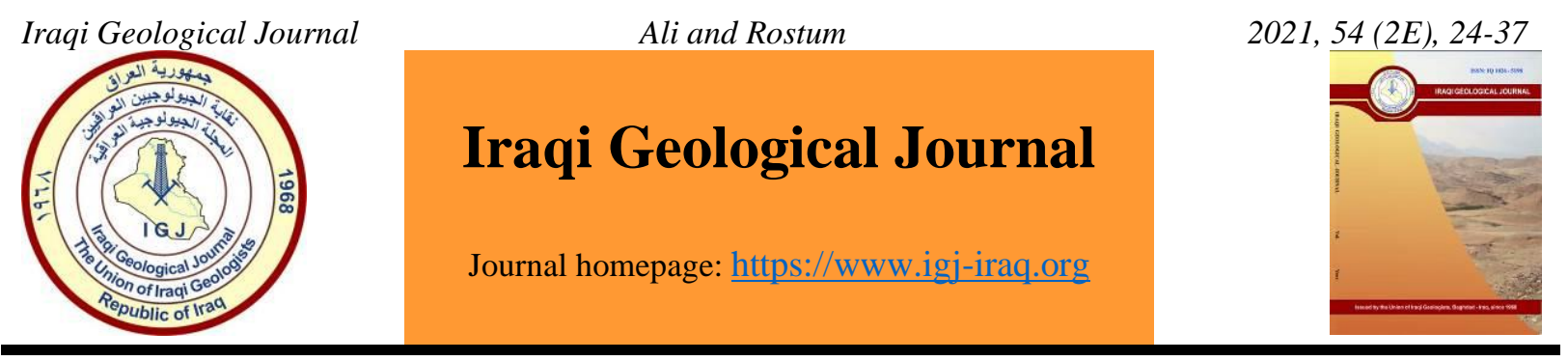

\title{
Petrography and Geochemistry of Gabbroic Rock from the Penjwin Ophiolite, Kurdistan Region, Northeastern Iraq
}

\author{
Sarmad A. Ali ${ }^{1,2, *}$ and Zaman Rostum ${ }^{1}$ \\ Department of Applied Geology, College of Science, Kirkuk University, Iraq \\ 2 School of Earth, Atmospheric and Life Sciences, University of Wollongong, Australia \\ * Correspondence: sarmad@uokirkuk.edu.iq
}

Received: 8 July 2021; Accepted: 9 August 2021; Published: 30 November 2021

\begin{abstract}
The gabbroic rocks as a part of Zagros ophiolite are exposed in northeastern Iraq, Penjwin area. These rocks with granular to ophitic textures are widely distributed without metamorphic halos. The main minerals are plagioclase (An ${ }_{90-99}$ ), olivine, clinopyroxene ( $\mathrm{Wo}_{27-47} \mathrm{En}_{45-64} \mathrm{Fs}_{8-14}$ ) and orthopyroxene ( $\mathrm{Wo}_{2} \mathrm{En}_{78} \mathrm{Fs}_{20}$ ) respectively based on the abundances. The major elements show a broad range of compositional variations, with $\mathrm{SiO}_{2}$ (46.2-50.9 wt. $\%$ ), and low concentrations $\mathrm{Na}_{2} \mathrm{O}\left(0.15-0.62\right.$ wt. \%), $\mathrm{K}_{2} \mathrm{O}\left(0.01-0.03\right.$ wt. \%) and $\mathrm{TiO}_{2}(0.06-0.2)$ and high concentrations, $\mathrm{Al}_{2} \mathrm{O}_{3}\left(6.4-19.75\right.$ wt. \%), total $\mathrm{Fe}_{2} \mathrm{O}_{3}$ (6.29-11.6 wt. \%), $\mathrm{MgO}$ (9.63-24.5 wt. \%), $\mathrm{CaO}(8.02-18$ wt. \%) and low alkali contents $\left(\mathrm{Na}_{2} \mathrm{O}+\mathrm{K}_{2} \mathrm{O}=0.16-0.65\right.$ wt. \%). On Ti-V diagram, all of the gabbroic samples have Ti/V less than 10 and consequently fall in the low Ti- Island arc tholeitic. Whole rocks chemistry shows a depletion of High field strength elements in comparison with the primitive mantle with an arched upward rare earth elements pattern, characterized by light rare earth elements depletion $(\mathrm{La} \mathrm{N} / \mathrm{Sm} \mathrm{N}=0.05-0.8)$ and enrichment in the High field strength elements. Whole rocks chemistry, mineral paragenesis and chemistry of these rocks are more consistent with tholeiitic magma series. Based on our findings in this research, the primary magma has been produced from the depleted mantle with a high degree of partial melting.
\end{abstract}

Keywords: Penjwin ophiolite complex; Zagros sutures zone; Neotethys; Suprasubduction zone; Gabbro

\section{Introduction}

The Zagros orogenic belt is located in the central Alpine-Himalayan orogenic system, this orogenic belt is estimated to be around $2000 \mathrm{~km}$ long, this was recorded depend on the geophysical and geochemical data (Alavi, 1994; Agard et al., 2005; Mohajjel and Ferguson, 2014; Shafaii Moghadam and Stern, 2015; Moghadam et al., 2019; Azizi and Moineraziri, 2009). Several magmatic activities have been recorded along Zagros orogenic belt including intra-oceanic arc trench systems during the late Mesozoic and the Paleogene. This led to formation of many ophiolites on the boundary between the Arabian and the Eurasian continental plates. The Iraqi Zagros ophiolites from southeast to the northwest is Penjwin, Mawat, Bulfat, Pushtashan and Hasanbag (Ali et al., 2019). The Penjwin ophiolite complex is part of the Penjwin Walash Zone in Iraq, this zone is about $\sim 16 \mathrm{~km}$ long and $\sim 5 \mathrm{~km}$ wide (Mahammod,1978) that trends northwest-southeast parallel to the regional structures and continues into the Iranian Zagros segment and consists of three thrust sheets: the structurally lowest Naopurdan, the middle Walash and the upper Qandil (Ali et al., 2012, 2013, 2014, 2016, 2019, Aswad et al., 2011, 2013,

DOI: $10.46717 /$ igj.54.2E.3Ms-2021-11-19 
Mohammad et al., 2014, 2017). The age 93.8 0.7 Ma of Penjwin ophiolite complex has been determined by Abdulla (2015) and Aziz et al. (2021) by using zircon U-Pb dating of plagiogranite rock. The Penjwin ophiolite in Iraq is a remnant of the late Cretaceous suprasubduction zone (SSZ) oceanic lithosphere that was developed in Southern Neotethys (Aswad, 1999, Abdulla, 2015, Ali et al., 2019). Gabbroic rocks are the main components of the Penjwin ophiolite complex. Study this type of rocks is crucial to determine the petrogenesis and evolution of ophiolites (Moore et al., 1971; Coogan et al., 2000; Furnes et al., 2020). Study area is located in Iraqi Zagros Thrust Zone that extends northwest-southeast from eastern Turkey through northern and northeastern Iraqi-Iranian border into northern Oman (Jassim and Goff, 2006; Moghadam and Stern, 2011, Ali et al., 2012, 2013, 2014, 2016, 2019, Znad et al., 2020, Aziz et al., 2021), which is also considered as a part of the main Zagros Orogenic Belt (Fig.1a). This orogenic belt is part of the Alpine-Himalayan Orogenic Belt which is one of the most comprehensive ophiolite depositories in earth's history. (Alavi, 1994; Talbot and Alavi, 1996; Stampfli and Borel, 2002; Ali et al., 2012, 2013, 2014, 2016, 2019). Moghadam and Stern (2011) was divided these ophiolites into two groups (Fig.1b).

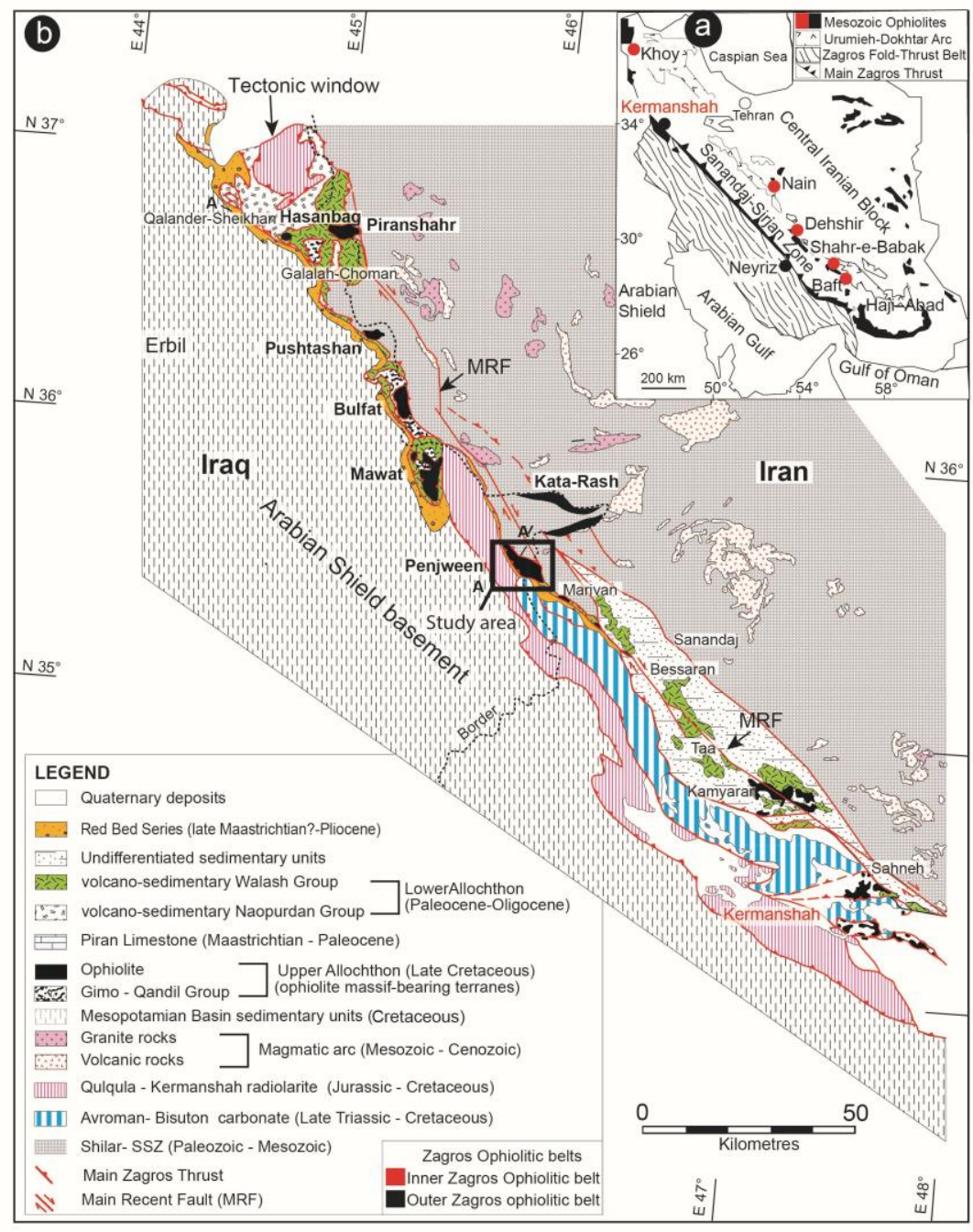

Fig.1. Geological maps of the Zagros thrust zone along the Iraq-Iran border, showing the location and tectonic subdivision of the study area (after Ali et al., 2019) 
The first group is located between the Sanandaj-Sirjan Zone and Central Iran consists of Khoy-Nain-Dehshir -Baft and is called Inner Zagros Ophiolitic Belt (Fig.1a), whereas the second group is located between the Sanandaj-Sirjan and Zagros thrust zone consists of Hasanbag-Pushtashan-Bulfat-Mawat-Penjween-Kermanshah-Neyriz-Haji Abad and is called Outer Zagros Ophiolitic Belt (Fig. 1b) (Moghadam and Stern, 2011; Ali et al., 2019). Due to lack of information about the gabbroic rocks in the Penjwin ophiolite complex, apart from only two papers by (Al- Hassan, 1975 and 1987, Al-Hassan and Hubbard, 1985). In this paper we present new field observations, petrographic, and geochemistry data from gabbroic rocks in the Penjwin ophiolite complex to interpret the genesis of these gabbroic rocks within the Penjwin ophiolite complex, which can provide an important key to understanding the genesis and emplacement of Tethyan ophiolites.

\section{Geological Setting}

The Penjwin ophiolite complex is situated in the northeastern of Iraqi Zagros Thrust Zone of about 60 kilometers east of Sulaimaniya city and southwest of Penjwin town about 4 kilometers, Kurdistan region between Latitudes $\left(35^{\circ} 35^{\prime}-35^{\circ} 38^{\prime} \mathrm{N}\right)$ and Longitudes $\left(45^{\circ} 52^{\prime}-46^{\circ} 00^{\prime} \mathrm{E}\right)$ (Figs. 1 and 2). The Penjwin igneous complex is one of five ophiolites found along the Iraqi Zagros thrust zone, it covers a large area estimated at about $\left(35 \mathrm{~km}^{2}\right)$ on the Iraqi side while the majority is extended to the Iranian side (Mahmmod, 1978; Al Hassan, 1982; Mohammad, 2008, Ali et al., 2019). This ophiolite is located between Red Bed and/or the Qulqula Formation and Gimo-Qandil Group (Figs. 1b and 2) and surrounded from the east by a Gimo-Qandil unit which is mostly consists of phyllite and calc-schist, whereas from the west by the Red Bed Series which consists of Cenozoic clastic by a high-angle reverse fault with the age of Miocene-Pliocene (Al Hassan and Hubbard, 1985; Jassim and Goff, 2006). According to Al- Hassan and Habbard, (1985) the Penjwin igneous complex contains mantle sequences (peridotite include harzburgite, dunite and lherzolite) on the bottom and oceanic crustal sequences accumulate gabbros, dykes of diorite and volcanic rocks on the top. In contrast, and depending on Ali et al., (2019) and Mohammad et al., (2020) and the current study fieldwork, it is very clear that the stratigraphy sequence of the Penjwin ophiolite complex from the top is ultramafic (peridotite include harzburgite, dunite and lherzolite) rocks overlain the gabbroic rocks (lower layered gabbro and upper gabbros) and the sheeted dykes in bottom. The conclusion of the geological of the Penjween ophiolite can be observed in new a geological map for the study area (Fig.2). It also supported by a stratigraphic column and cross-section of the Penjwin ophiolite complex modified by (Ali et al., 2019) (Figs. 2 and 3). According to (Aswad, (1999) this ophiolite located in oceanic supra-subduction ophiolite.

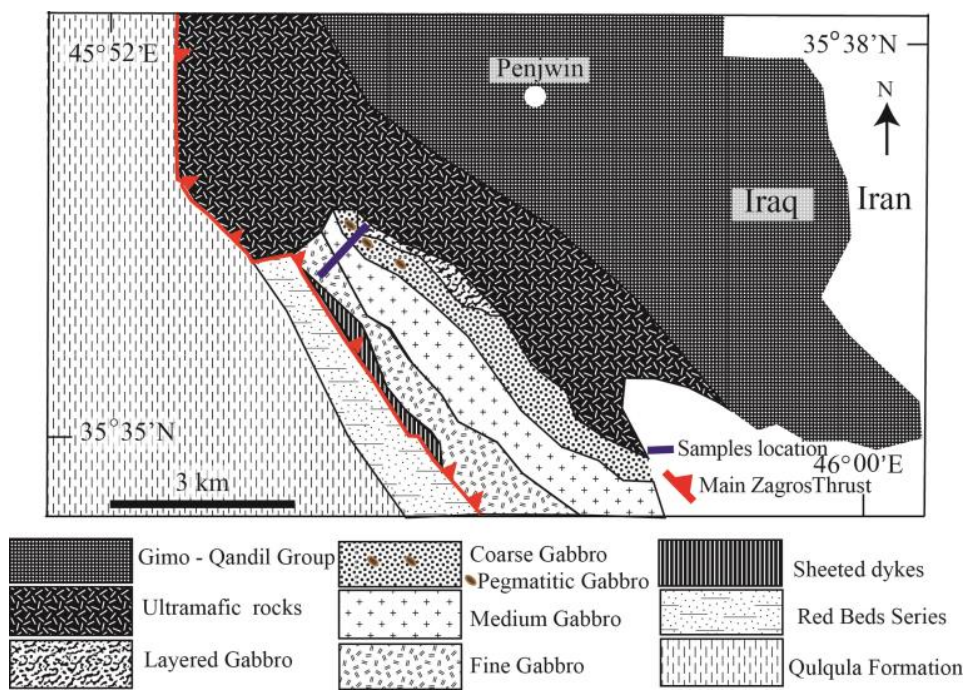

Fig.2. Geologic map of Penjwin Ophiolite complex showing the locations of the gabbroic samples 


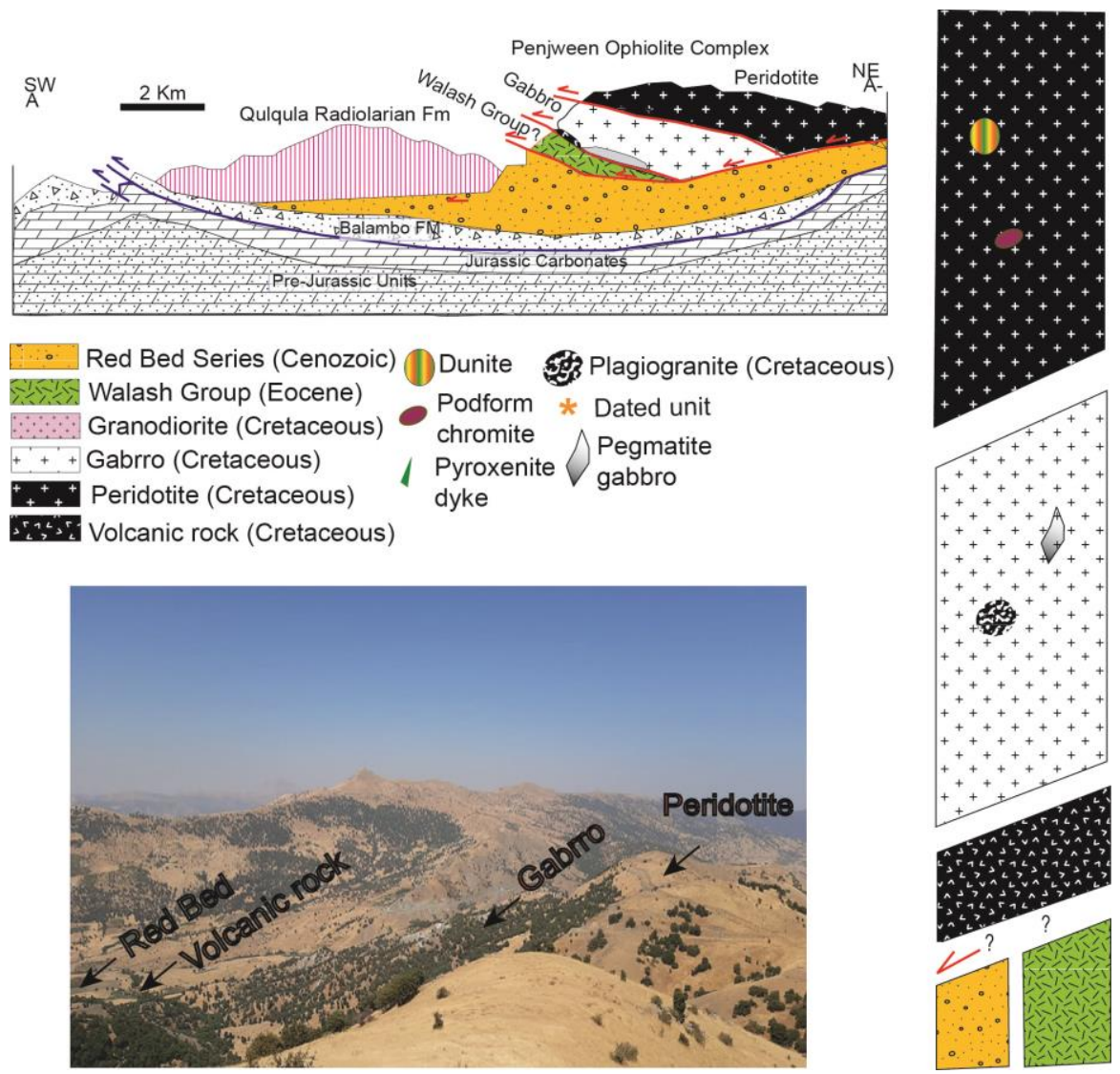

Fig. 3. Stratigraphic column, cross-section and google earth photo of Penjween ophiolite (modified from Ali et al., 2019 and Mohammad et al., 2021)

\section{Analytical Techniques}

\subsection{Electron Microprobe Mineral Analyses}

Mineral chemistry was determined using a Scanning Electron Microscopy (SEM) in the Geometallurgy Laboratory Freiberg, Technische Universitat Bergakademie Freiberg, based on a scanning electron microscope SEM Quanta 650 - FEG - MLA (Division of Economic Geology and Petrology, Institute of Mineralogy, TU Bergakademie Freiberg, Germany), equipped with Bruker Dual $X$ - Flash spectrometer for energy dispersive spectrometry (EDS) analyses were applied to complete rock thin sections. Details of the analytical protocol and the instrumental conditions can be found in (Schulz et al., 2020).

\subsection{Major Element and Trace Elements Analyses}

Whole-rock geochemistry (major, trace and rare earth elements) and Loss-on-ignition were analyzed in the Australian Laboratory Services (ALS) Laboratory Group SL Camas Spain, by ICP-MS, ICP-AES and with the Lithium Borate fusion method as a whole rock package encoding (ME-ICP06), (ME-MS 81D), (OA-GRA05) and (TOT-ICP06).

\subsection{Petrography and mineral chemistry}

Petrographic studies were undertaken on 20 thin sections of Penjwin gabbroic rocks. The Penjwin gabbroic rocks are mostly fresh and medium to coarse grained. Its essential mineral composition includes $15-60 \%$ plagioclase, $1-25 \%$ olivine, $30-70 \%$ clinopyroxene, orthopyroxene $1-12 \%$, and 1-2\% amphiboles and iron oxides $2-10 \%$, while the secondary minerals are secondary amphibole (mostly 
tremolite and actinolite), chlorite and sericite. The rocks show dominantly intergranular, ophitic, subophitic and poikilitic textures (Fig. 4). Plagioclase is mostly $\mathrm{Ca}$ rich (anorthite and Bytownite) $\left(\mathrm{An}_{90-99}\right)$ (Table 1 and Fig. 5A). Olivine is the most abundant mafic minerals in the lower gabbroic part and is rounded to sub-rounded with a composition of $\mathrm{F}_{78}$. Unusually, olivine minerals are fresh in most gabbroic rocks, but some olivine crystals show alteration (Fig. 4B and Table 2). Orthopyroxene of enstatite composition $\mathrm{Wo}_{2} \mathrm{En}_{78} \mathrm{Fs}_{20}$, formed between the plagioclase and olivine, while clinopyroxene is mostly augite and diopside $\mathrm{Wo}_{27-47} \mathrm{En}_{45-64} \mathrm{Fs}_{8-14}$ (Figs. 4A, B, C, E, 5B and Table 3). Exsolution lamellae of clinopyroxene are observed in the orthopyroxene (Fig. 4 E). In addition, the presence of pigeonite (FG6-Pxmat4, FG6-Pxmat3) in an igneous rock thus provides evidence for the crystallization temperature of the magma, and hence indirectly for the water content of that magma. Amphibole is one of the primary minerals that may be present in a small amount in gabbroic rocks. However, most of them are secondary amphibole minerals with a small amount of primary amphibole (Mg-hornblende) (Figs. 4E, 5C and Table 5). Fe-Ti oxides comprise 1-10\% of the olivine gabbro samples. Ilmenite is the most common oxide and forms irregular interstitial grains or inclusions in olivine and amphibole. Ti magnetite occurs along the cleavages and cracks of olivine (Figs.4F, 5D and Table 4).
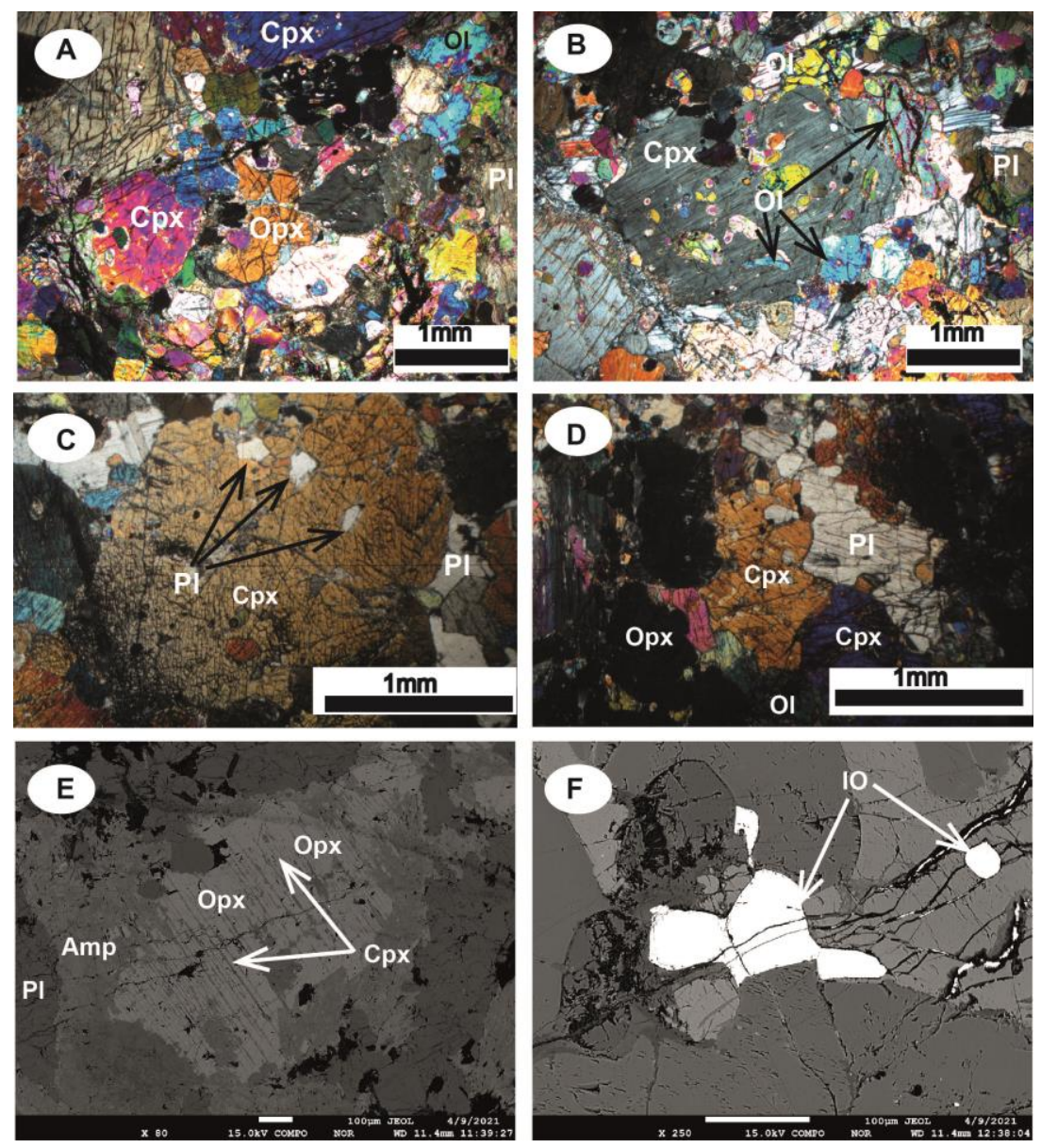

Fig. 4. Photomicrographs of gabbroic rock from Penjwin ophiolite complex. (A) Granular texture; crossed polarizers (XPL); sample FG6; (B) Poikilitic texture, XPL; sample FG6; (C) Subophitic and ophitic texture, showing clinopyroxene partly and completely surrounding plagioclase grains; (XPL) sample FG5;(D) Intergranular texture; (XPL); sample FG5; (E) Backscatter image (BSE) of Exsolution lamellar texture shows clinopyroxene lamellar in host orthopyroxene; sample LG2; (F) Backscatter image (BSE) of iron oxide magnetite and ilmenite sample FG6. $\mathrm{Pl}=$ Plagioclase; $\mathrm{Cpx}=$ Clinopyroxene; Opx=Orthopyroxene; Amph = Amphibole; $\mathrm{IO}=$ Iron oxide . 

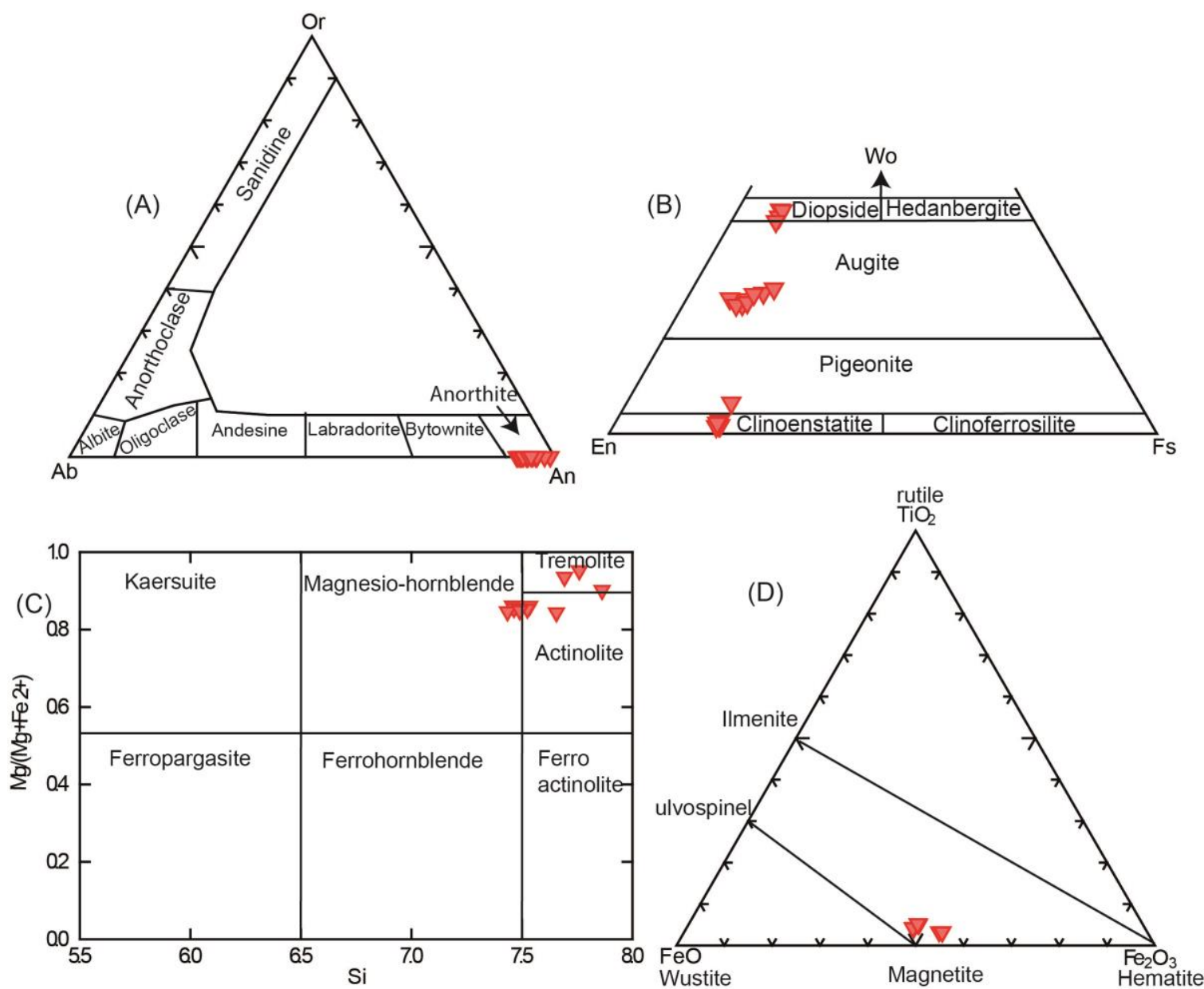

Fig. 5. (A) Compositions of plagioclase from Penjwin gabbroic rocks plotted on the Ab-An-Or diagram; (B) Composition of pyroxenes from Penjwin gabbroic rocks in the Wo-En-Fs diagram; (C) Composition diagrams of amphiboles from Penjwin gabbroic rocks; (D) Composition diagrams of iron oxides from Penjwin gabbroic rocks

\section{Results and Interpretations}

\subsection{Whole Rock Geochemistry}

The major, trace and rare earth element contents of the studied Penjwin gabbroic samples are listed in Table 6. The samples show a wide range of compositional variations, with $\mathrm{SiO}_{2}$ (46.2-50.9 wt. \%), and low concentrations $\mathrm{Na}_{2} \mathrm{O}\left(0.15-0.62\right.$ wt. \%), $\mathrm{K}_{2} \mathrm{O}\left(0.01-0.03\right.$ wt. \%) and $\mathrm{TiO}_{2}(0.06-0.2)$ and high concentrations, $\mathrm{Al}_{2} \mathrm{O}_{3}$ (6.4-19.75 wt. \%), $\mathrm{Fe}_{2} \mathrm{O}_{3}$ (6.29-11.6 wt. \%), $\mathrm{MgO}$ (9.63-24.5 wt. \%), $\mathrm{CaO}$ (8.02-18 wt. \%) and low alkali contents $(\mathrm{Na} 2 \mathrm{O}+\mathrm{K} 2 \mathrm{O}=0.16-0.65$ wt. \%). In the Middlemost 1985 diagrams $\left(\mathrm{SiO}_{2}\right.$ vs. $\left.\mathrm{Na}_{2} \mathrm{O}+\mathrm{K}_{2} \mathrm{O}\right)$ the rocks are totally plotted in the gabbro field (Fig.6 A). According to the Shervais (1982) Ti-V diagram, all of the gabbroic samples have Ti/V from less than 10 and consequently fall in the low Ti- Island arc tholeiitic (IAT) (Fig.6 B). Moreover, and depend on (AFM) discrimination diagram (after Irvine and Baragar, 1971) the Penjwin gabbroic rocks belong to the tholeiitic affinities (Fig. 6C). Whereas, all Penjwin gabbroic rocks fall in the ocean ridge field on MgO-FeO*- $\mathrm{Al}_{2} \mathrm{O}_{3}$ ternary discrimination diagram of Pearce and Gale, (1977) (Fig.6 D). 
Table 1. Selective representative of electron microprobe analyses of plagioclase for gabbroic rock of Penjwin ophiolite complex

\begin{tabular}{|c|c|c|c|c|c|c|c|c|}
\hline sample & LG2-PI1-1 & LG2-PI1-2 & LG2-PI1-3 & FG6-PI2-1 & FG6-PI2-3 & FG6-PI2-5 & FG6-PI1-4 & FG6-PI1-6 \\
\hline $\mathrm{SiO}_{2}$ & 44.98 & 45.66 & 45.65 & 44.53 & 44.03 & 44.28 & 44.68 & 45.67 \\
\hline $\mathrm{TiO}_{2}$ & 0.00 & 0.00 & 0.00 & 0.00 & 0.03 & 0.02 & 0.02 & 0.00 \\
\hline $\mathrm{Al}_{2} \mathrm{O}_{3}$ & 34.62 & 34.22 & 34.12 & 34.40 & 33.89 & 34.30 & 34.03 & 33.77 \\
\hline $\mathrm{Cr}_{2} \mathrm{O}_{3}$ & 0.00 & 0.00 & 0.00 & 0.00 & 0.00 & 0.00 & 0.02 & 0.00 \\
\hline $\mathrm{Fe}_{2} \mathrm{O}_{3}$ & 0.00 & 0.00 & 0.00 & 0.00 & 0.00 & 0.00 & 0.00 & 0.00 \\
\hline $\mathrm{FeO}$ & 0.05 & 0.05 & 0.04 & 0.33 & 0.26 & 0.30 & 0.31 & 0.30 \\
\hline $\mathrm{MnO}$ & 0.01 & 0.02 & 0.01 & 0.01 & 0.01 & 0.01 & 0.01 & 0.00 \\
\hline $\mathrm{MgO}$ & 0.01 & 0.00 & 0.07 & 0.03 & 0.00 & 0.00 & 0.02 & 0.05 \\
\hline $\mathrm{CaO}$ & 19.24 & 18.93 & 19.08 & 18.75 & 18.52 & 18.68 & 18.55 & 18.19 \\
\hline $\mathrm{BaO}$ & 0.00 & 0.00 & 0.00 & 0.00 & 0.00 & 0.00 & 0.00 & 0.00 \\
\hline $\mathrm{Na}_{2} \mathrm{O}$ & 0.48 & 0.61 & 0.61 & 0.68 & 0.84 & 0.66 & 0.81 & 0.69 \\
\hline $\mathrm{K}_{2} \mathrm{O}$ & 0.00 & 0.01 & 0.01 & 0.00 & 0.01 & 0.00 & 0.00 & 0.00 \\
\hline Total & 99.37 & 99.50 & 99.58 & 98.74 & 97.58 & 98.26 & 98.45 & 98.67 \\
\hline $\mathrm{Si}$ & 2.09 & 2.12 & 2.12 & 2.08 & 2.08 & 2.08 & 2.10 & 2.14 \\
\hline $\mathrm{Ti}$ & 0.00 & 0.00 & 0.00 & 0.00 & 0.00 & 0.00 & 0.00 & 0.00 \\
\hline $\mathrm{Al}$ & 1.90 & 1.88 & 1.87 & 1.90 & 1.89 & 1.90 & 1.88 & 1.87 \\
\hline $\mathrm{Cr}$ & 0.00 & 0.00 & 0.00 & 0.00 & 0.00 & 0.00 & 0.00 & 0.00 \\
\hline $\mathrm{Fe}^{+3}$ & 0.00 & 0.00 & 0.00 & 0.00 & 0.00 & 0.00 & 0.00 & 0.00 \\
\hline $\mathrm{Fe}^{+2}$ & 0.00 & 0.00 & 0.00 & 0.01 & 0.01 & 0.01 & 0.01 & 0.01 \\
\hline $\mathrm{Mn}$ & 0.00 & 0.00 & 0.00 & 0.00 & 0.00 & 0.00 & 0.00 & 0.00 \\
\hline $\mathrm{Mg}$ & 0.00 & 0.00 & 0.00 & 0.00 & 0.00 & 0.00 & 0.00 & 0.00 \\
\hline $\mathrm{Ca}$ & 0.96 & 0.94 & 0.95 & 0.94 & 0.94 & 0.94 & 0.93 & 0.91 \\
\hline $\mathrm{Ba}$ & 0.00 & 0.00 & 0.00 & 0.00 & 0.00 & 0.00 & 0.00 & 0.00 \\
\hline $\mathrm{Na}$ & 0.04 & 0.05 & 0.05 & 0.06 & 0.08 & 0.06 & 0.07 & 0.06 \\
\hline $\mathrm{K}$ & 0.00 & 0.00 & 0.00 & 0.00 & 0.00 & 0.00 & 0.00 & 0.00 \\
\hline tot. cat. & 5.00 & 5.00 & 5.00 & 5.00 & 5.00 & 5.00 & 5.00 & 5.00 \\
\hline tot.oxy. & 8.02 & 8.03 & 8.03 & 8.00 & 7.99 & 8.00 & 8.00 & 8.04 \\
\hline An & 95.72 & 94.45 & 94.50 & 93.84 & 92.39 & 93.97 & 92.68 & 93.58 \\
\hline $\mathrm{Ab}$ & 4.28 & 5.51 & 5.47 & 6.16 & 7.58 & 6.03 & 7.30 & 6.40 \\
\hline Or & 0.00 & 0.04 & 0.04 & 0.00 & 0.03 & 0.00 & 0.02 & 0.02 \\
\hline
\end{tabular}

Table 2. Selective representative electron microprobe analyses of olivine for gabbroic rock of Penjwin ophiolite complex

\begin{tabular}{|c|c|c|c|c|}
\hline Sample & FG6-Ol1 & FG6-O12 & FG6-Ol3 & FG6-O14 \\
\hline $\mathrm{SiO}_{2}$ & 39.38 & 38.92 & 38.87 & 38.63 \\
\hline $\mathrm{TiO}_{2}$ & 0.01 & 0.00 & 0.00 & 0.01 \\
\hline $\mathrm{Al}_{2} \mathrm{O}_{3}$ & 0.01 & 0.00 & 0.00 & 0.00 \\
\hline $\mathrm{Cr}_{2} \mathrm{O}_{3}$ & 0.02 & 0.03 & 0.01 & 0.02 \\
\hline $\mathrm{Fe}_{2} \mathrm{O}_{3}$ & 0.00 & 0.00 & 0.01 & 0.03 \\
\hline $\mathrm{FeO}$ & 19.31 & 19.78 & 19.35 & 19.61 \\
\hline $\mathrm{MnO}$ & 0.26 & 0.24 & 0.23 & 0.23 \\
\hline $\mathrm{MgO}$ & 41.05 & 40.97 & 41.16 & 40.70 \\
\hline $\mathrm{CaO}$ & 0.04 & 0.02 & 0.01 & 0.03 \\
\hline total & 100.08 & 99.95 & 99.64 & 99.26 \\
\hline $\mathrm{Si}$ & 1.01 & 1.00 & 1.00 & 1.00 \\
\hline $\mathrm{Ti}$ & 0.00 & 0.00 & 0.00 & 0.00 \\
\hline $\mathrm{Al}$ & 0.00 & 0.00 & 0.00 & 0.00 \\
\hline $\mathrm{Cr}$ & 0.00 & 0.00 & 0.00 & 0.00 \\
\hline $\mathrm{Fe}^{+3}$ & 0.00 & 0.00 & 0.00 & 0.00 \\
\hline $\mathrm{Fe}^{+2}$ & 0.41 & 0.42 & 0.42 & 0.42 \\
\hline $\mathrm{Mn}$ & 0.01 & 0.01 & 0.00 & 0.01 \\
\hline $\mathrm{Mg}$ & 1.57 & 1.57 & 1.58 & 1.57 \\
\hline $\mathrm{Ca}$ & 0.00 & 0.00 & 0.00 & 0.00 \\
\hline tot. cat. & 3.00 & 3.00 & 3.00 & 3.00 \\
\hline tot.oxy. & 4.01 & 4.00 & 4.00 & 4.00 \\
\hline $\mathrm{Te}$ & 0.28 & 0.26 & 0.25 & 0.25 \\
\hline Fo & 78.86 & 78.46 & 78.92 & 78.47 \\
\hline $\mathrm{Fa}$ & 20.81 & 21.25 & 20.82 & 21.24 \\
\hline
\end{tabular}


Table 3. Selective representative of electron microprobe of pyroxene for gabbroic rock of Penjwin ophiolite complex

\begin{tabular}{lcccccccc}
\hline Sample & FG6Pxm2 & FG6Px32 & FG6Px25 & FG6Px22 & FG6Pxmat2 & FG6Pxmat4 & FG6Pxmat3 & FG6Pxm1 \\
\hline $\mathrm{SiO}_{2}$ & 51.84 & 54.63 & 55.32 & 54.40 & 53.30 & 55.39 & 53.56 & 51.84 \\
$\mathrm{TiO}_{2}$ & 0.36 & 0.21 & 0.14 & 0.10 & 0.34 & 0.15 & 0.16 & 0.36 \\
$\mathrm{Al}_{2} \mathrm{O}_{3}$ & 2.10 & 1.50 & 1.56 & 1.56 & 2.02 & 1.69 & 1.81 & 2.10 \\
$\mathrm{Cr}_{2} \mathrm{O}_{3}$ & 0.40 & 0.29 & 0.28 & 0.33 & 0.40 & 0.39 & 0.33 & 0.40 \\
$\mathrm{Fe}_{2} \mathrm{O}_{3}$ & 1.25 & 0.55 & 0.00 & 0.53 & 0.07 & 0.00 & 1.94 & 1.25 \\
$\mathrm{FeO}$ & 3.43 & 12.00 & 12.60 & 11.50 & 8.98 & 13.41 & 11.27 & 3.43 \\
$\mathrm{MnO}$ & 0.14 & 0.26 & 0.24 & 0.23 & 0.17 & 0.25 & 0.25 & 0.14 \\
$\mathrm{MgO}$ & 16.06 & 29.11 & 29.14 & 28.44 & 19.63 & 28.67 & 26.92 & 16.06 \\
$\mathrm{CaO}$ & 23.08 & 0.99 & 0.87 & 2.05 & 15.10 & 0.64 & 3.57 & 23.08 \\
$\mathrm{Na} 2 \mathrm{O}$ & 0.12 & 0.02 & 0.01 & 0.02 & 0.12 & 0.01 & 0.02 & 0.12 \\
$\mathrm{Total}$ & 98.76 & 99.56 & 100.16 & 99.16 & 100.13 & 100.60 & 99.84 & 98.76 \\
$\mathrm{Si}$ & 1.93 & 1.95 & 1.96 & 1.95 & 1.94 & 1.97 & 1.93 & 1.93 \\
$\mathrm{Ti}$ & 0.01 & 0.01 & 0.00 & 0.00 & 0.01 & 0.00 & 0.00 & 0.01 \\
$\mathrm{Al}$ & 0.09 & 0.06 & 0.07 & 0.07 & 0.09 & 0.07 & 0.08 & 0.09 \\
$\mathrm{Cr}$ & 0.01 & 0.01 & 0.01 & 0.01 & 0.01 & 0.01 & 0.01 & 0.01 \\
$\mathrm{Fe}$ & 0.03 & 0.01 & 0.00 & 0.01 & 0.00 & 0.00 & 0.05 & 0.03 \\
$\mathrm{Fe}+2$ & 0.11 & 0.36 & 0.37 & 0.35 & 0.27 & 0.40 & 0.34 & 0.11 \\
$\mathrm{Mn}$ & 0.00 & 0.01 & 0.01 & 0.01 & 0.01 & 0.01 & 0.01 & 0.00 \\
$\mathrm{Mg}$ & 0.89 & 1.55 & 1.54 & 1.52 & 1.07 & 1.52 & 1.44 & 0.89 \\
$\mathrm{Ca}$ & 0.92 & 0.04 & 0.03 & 0.08 & 0.59 & 0.02 & 0.14 & 0.92 \\
$\mathrm{Na}$ & 0.01 & 0.00 & 0.00 & 0.00 & 0.01 & 0.00 & 0.00 & 0.01 \\
tot. cat. & 4.00 & 4.00 & 4.00 & 4.00 & 4.00 & 4.00 & 4.00 & 4.00 \\
tot. oxy. & 6.00 & 6.00 & 6.00 & 6.00 & 6.00 & 6.01 & 6.00 & 6.00 \\
$\mathrm{XWo}$ & 47.11 & 1.93 & 1.70 & 4.03 & 30.53 & 1.26 & 6.97 & 47.11 \\
$\mathrm{Xen}$ & 45.63 & 79.03 & 79.10 & 77.63 & 55.20 & 78.21 & 73.17 & 45.63 \\
$\mathrm{XFs}$ & 7.26 & 19.04 & 19.19 & 18.34 & 14.28 & 20.53 & 19.86 & 7.26 \\
\hline
\end{tabular}

Table 4. Selective representative of electron microprobe analyses of amphibole for gabbroic rock of Penjwin ophiolite complex

\begin{tabular}{|c|c|c|c|c|c|c|c|}
\hline Sample & LG2-Am1-1 & LG2-Am1-3 & LG2-Am1-7 & LG2-Am2-3 & LG2-Am1-2 & LG2-Am1-4 & LG2-Am2-1 \\
\hline $\mathrm{SiO}_{2}$ & 53.66 & 53.24 & 52.59 & 56.07 & 52.73 & 52.17 & 56.65 \\
\hline $\mathrm{TiO}_{2}$ & 0.16 & 0.15 & 0.20 & 0.08 & 0.18 & 0.16 & 0.06 \\
\hline $\mathrm{Al}_{2} \mathrm{O}_{3}$ & 1.72 & 1.95 & 2.12 & 2.04 & 2.34 & 2.22 & 1.32 \\
\hline $\mathrm{Cr}_{2} \mathrm{O}_{3}$ & 0.20 & 0.19 & 0.25 & 0.10 & 0.18 & 0.28 & 0.16 \\
\hline $\mathrm{Fe}_{2} \mathrm{O}_{3}$ & 0.00 & 0.00 & 0.00 & 3.71 & 0.00 & 0.00 & 2.33 \\
\hline $\mathrm{FeO}$ & 4.59 & 4.82 & 4.55 & 1.86 & 4.80 & 5.09 & 3.93 \\
\hline $\mathrm{MnO}$ & 0.13 & 0.16 & 0.12 & 0.20 & 0.17 & 0.17 & 0.21 \\
\hline $\mathrm{MgO}$ & 15.87 & 15.67 & 15.86 & 20.67 & 15.84 & 15.71 & 20.16 \\
\hline $\mathrm{CaO}$ & 23.49 & 23.03 & 23.29 & 12.45 & 22.85 & 22.86 & 12.63 \\
\hline $\mathrm{Na}_{2} \mathrm{O}$ & 0.19 & 0.17 & 0.20 & 0.15 & 0.16 & 0.22 & 0.11 \\
\hline $\mathrm{K}_{2} \mathrm{O}$ & 0.00 & 0.00 & 0.00 & 0.00 & 0.00 & 0.00 & 0.00 \\
\hline $\mathrm{ZrO}_{2}$ & 0.00 & 0.00 & 0.00 & 0.00 & 0.00 & 0.00 & 0.00 \\
\hline Total & 100.00 & 99.38 & 99.17 & 97.33 & 99.24 & 98.89 & 97.57 \\
\hline $\mathrm{Si}$ & 7.54 & 7.52 & 7.46 & 7.76 & 7.46 & 7.43 & 7.86 \\
\hline $\mathrm{Ti}$ & 0.02 & 0.02 & 0.02 & 0.01 & 0.02 & 0.02 & 0.01 \\
\hline $\mathrm{Al}$ & 0.28 & 0.32 & 0.35 & 0.33 & 0.39 & 0.37 & 0.22 \\
\hline $\mathrm{Cr}$ & 0.02 & 0.02 & 0.03 & 0.01 & 0.02 & 0.03 & 0.02 \\
\hline $\mathrm{Fe}^{+3}$ & 0.00 & 0.00 & 0.00 & 0.39 & 0.00 & 0.00 & 0.24 \\
\hline $\mathrm{Fe}^{+2}$ & 0.54 & 0.57 & 0.54 & 0.21 & 0.57 & 0.61 & 0.46 \\
\hline Mn & 0.01 & 0.02 & 0.01 & 0.02 & 0.02 & 0.02 & 0.02 \\
\hline $\mathrm{Mg}$ & 3.32 & 3.30 & 3.35 & 4.26 & 3.34 & 3.34 & 4.17 \\
\hline $\mathrm{Ca}$ & 3.53 & 3.49 & 3.54 & 1.85 & 3.46 & 3.49 & 1.88 \\
\hline $\mathrm{Na}$ & 0.05 & 0.05 & 0.06 & 0.04 & 0.04 & 0.06 & 0.03 \\
\hline $\mathrm{K}$ & 0.00 & 0.00 & 0.00 & 0.00 & 0.00 & 0.00 & 0.00 \\
\hline $\mathrm{Zr}$ & 0.00 & 0.00 & 0.00 & 0.00 & 0.00 & 0.00 & 0.00 \\
\hline TOTAL & 15.32 & 15.31 & 15.36 & 14.89 & 15.33 & 15.38 & 14.91 \\
\hline Type & Actin & Actin & Eden & Trem & Eden & Actin & Trem \\
\hline $\mathrm{Mg} /\left(\mathrm{Mg}+\mathrm{Fe}^{+2}\right)$ & 0.86 & 0.85 & 0.86 & 0.95 & 0.85 & 0.85 & 0.90 \\
\hline
\end{tabular}


Table 5. Selective representative of electron microprobe analyses of iron oxide for gabbroic rock of Penjwin ophiolite complex.

\begin{tabular}{lccccccccc}
\hline Sample & FG6-x1 & FG6-x4 & FG6-x7 & FG6-x8 & FG6-x5 & FG6-x2 & FG6-x9 & FG6-x6 & FG6-x3 \\
\hline $\mathrm{SiO}_{2}$ & 0.00 & 0.02 & 0.03 & 0.03 & 0.00 & 0.02 & 0.01 & 0.02 & 0.00 \\
$\mathrm{TiO}_{2}$ & 2.47 & 2.79 & 3.58 & 2.42 & 2.83 & 3.56 & 2.40 & 2.78 & 3.61 \\
$\mathrm{Al}_{2} \mathrm{O}_{3}$ & 5.48 & 8.31 & 6.97 & 5.90 & 8.49 & 7.12 & 5.58 & 8.58 & 7.28 \\
$\mathrm{Cr}_{2} \mathrm{O}_{3}$ & 17.02 & 22.12 & 20.05 & 17.65 & 22.17 & 20.11 & 17.14 & 22.26 & 20.43 \\
$\mathrm{Fe}_{2} \mathrm{O}_{3}$ & 40.25 & 30.98 & 32.91 & 39.30 & 31.16 & 33.59 & 40.17 & 30.97 & 33.19 \\
$\mathrm{FeO}$ & 31.86 & 31.48 & 32.25 & 32.07 & 31.62 & 32.62 & 31.91 & 31.73 & 32.59 \\
$\mathrm{MnO}$ & 0.38 & 0.48 & 0.43 & 0.37 & 0.47 & 0.39 & 0.40 & 0.45 & 0.41 \\
$\mathrm{MgO}$ & 1.23 & 1.87 & 1.69 & 1.21 & 1.93 & 1.70 & 1.19 & 1.89 & 1.80 \\
$\mathrm{CaO}$ & 0.03 & 0.00 & 0.00 & 0.00 & 0.02 & 0.02 & 0.00 & 0.00 & 0.01 \\
$\mathrm{ZnO}$ & 0.00 & 0.00 & 0.00 & 0.00 & 0.00 & 0.00 & 0.00 & 0.00 & 0.00 \\
$\mathrm{Total}$ & 98.72 & 98.04 & 97.91 & 98.96 & 98.70 & 99.13 & 98.80 & 98.67 & 99.32 \\
$\mathrm{Si}$ & 0.00 & 0.00 & 0.01 & 0.01 & 0.00 & 0.00 & 0.00 & 0.01 & 0.00 \\
$\mathrm{Ti}$ & 0.55 & 0.61 & 0.79 & 0.54 & 0.62 & 0.78 & 0.53 & 0.61 & 0.79 \\
$\mathrm{Al}$ & 1.92 & 2.86 & 2.42 & 2.05 & 2.90 & 2.44 & 1.95 & 2.93 & 2.49 \\
$\mathrm{Cr}$ & 3.99 & 5.10 & 4.67 & 4.12 & 5.08 & 4.63 & 4.02 & 5.10 & 4.69 \\
$\mathrm{Fe}$ & 8.99 & 6.80 & 7.30 & 8.73 & 6.79 & 7.36 & 8.96 & 6.75 & 7.25 \\
$\mathrm{Fe}$ & 7.90 & 7.68 & 7.95 & 7.92 & 7.66 & 7.94 & 7.91 & 7.69 & 7.91 \\
$\mathrm{Mn}$ & 0.10 & 0.12 & 0.11 & 0.09 & 0.11 & 0.10 & 0.10 & 0.11 & 0.10 \\
$\mathrm{Mg}$ & 0.54 & 0.81 & 0.74 & 0.53 & 0.84 & 0.74 & 0.53 & 0.81 & 0.78 \\
$\mathrm{Ca}$ & 0.01 & 0.00 & 0.00 & 0.00 & 0.01 & 0.00 & 0.00 & 0.00 & 0.00 \\
$\mathrm{Zn}$ & 0.00 & 0.00 & 0.00 & 0.00 & 0.00 & 0.00 & 0.00 & 0.00 & 0.00 \\
$\mathrm{Total}$ & 24 & 24 & 24 & 24 & 24 & 24 & 24 & 24 & 24 \\
\hline
\end{tabular}

Table 6. Whole rock geochemical analyses of Penjwin gabbroic with major (wt.\%) and trace element (ppm)

\begin{tabular}{|c|c|c|c|c|c|c|c|c|c|c|c|c|}
\hline Sample & LG1 & LG2 & LG3 & FG8 & FG5 & FG6 & PG1 & PG2 & PG6 & CG10 & G1 & G3 \\
\hline $\mathrm{SiO}_{2}$ & 47.4 & 49 & 50.9 & 47.7 & 49.5 & 46.2 & 47 & 47.9 & 46.9 & 48.4 & 49.5 & 48.5 \\
\hline $\mathrm{Al}_{2} \mathrm{O}_{3}$ & 13 & 12.9 & 6.42 & 19.75 & 6.4 & 6.79 & 17.5 & 17.25 & 18.15 & 16.95 & 16.5 & 15.35 \\
\hline $\mathrm{Fe}_{2} \mathrm{O}_{3}$ & 7.33 & 6.64 & 6.84 & 6.29 & 11.4 & 11.6 & 10.75 & 7.21 & 7.15 & 6.53 & 7.04 & 10.4 \\
\hline $\mathrm{CaO}$ & 15 & 14.45 & 18 & 14.85 & 9.16 & 8.02 & 13.05 & 15.05 & 14.65 & 13.5 & 15.15 & 13.85 \\
\hline $\mathrm{MgO}$ & 13.7 & 14.4 & 16.3 & 9.63 & 22.2 & 24.5 & 10.65 & 11.3 & 9.69 & 13.3 & 12.1 & 11.25 \\
\hline $\mathrm{Na}_{2} \mathrm{O}$ & 0.42 & 0.37 & 0.37 & 0.59 & 0.15 & 0.16 & 0.38 & 0.55 & 0.62 & 0.29 & 0.4 & 0.42 \\
\hline $\mathrm{K}_{2} \mathrm{O}$ & 0.03 & 0.03 & 0.01 & 0.03 & 0.01 & $<0.01$ & 0.02 & 0.01 & 0.02 & $<0.01$ & $<0.01$ & 0.01 \\
\hline $\mathrm{TiO}_{2}$ & 0.11 & 0.08 & 0.15 & 0.1 & 0.13 & 0.14 & 0.2 & 0.1 & 0.1 & 0.06 & 0.09 & 0.11 \\
\hline $\mathrm{MnO}$ & 0.14 & 0.14 & 0.14 & 0.12 & 0.19 & 0.17 & 0.16 & 0.13 & 0.13 & 0.13 & 0.14 & 0.18 \\
\hline LOI & 2.35 & 2.12 & 1.8 & 1.76 & 0.52 & 1.05 & 1.02 & 1.96 & 1.91 & 1.05 & 0.23 & 0.25 \\
\hline Total & 99.6 & 100.24 & 101.1 & 100.87 & 100 & 98.99 & 100.78 & 101.52 & 99.36 & 100.26 & 101.22 & 100.36 \\
\hline $\mathrm{Mg} \#$ & 78.73 & 81.12 & 82.52 & 75.20 & 79.41 & 80.71 & 66.24 & 75.64 & 72.86 & 80.14 & 77.30 & 68.18 \\
\hline $\mathrm{Ba}$ & 6.6 & 3.5 & 5.9 & 7.6 & 4.6 & 1.4 & 18 & 59 & 11.9 & 1.7 & 5.7 & 3.8 \\
\hline $\mathrm{Ce}$ & 0.5 & 0.4 & 0.4 & 0.9 & 0.3 & 0.3 & 0.2 & 0.3 & 0.5 & 0.1 & 0.2 & 0.4 \\
\hline $\mathrm{Cr}$ & 830 & 800 & 1370 & 280 & 2590 & 2740 & 270 & 290 & 250 & 300 & 420 & 200 \\
\hline Cs & 0.06 & 0.06 & 0.04 & 0.23 & 0.06 & 0.04 & 0.03 & 0.04 & 0.07 & 0.03 & 0.0 & 0.04 \\
\hline Dy & 0.52 & 0.41 & 0.97 & 0.44 & 0.49 & 0.47 & 0.32 & 0.45 & 0.43 & 0.26 & 0.47 & 0.37 \\
\hline Er & 0.36 & 0.26 & 0.64 & 0.29 & 0.37 & 0.34 & 0.21 & 0.3 & 0.3 & 0.18 & 0.33 & 0.25 \\
\hline $\mathrm{Eu}$ & 0.09 & 0.09 & 0.14 & 0.12 & 0.07 & 0.09 & 0.08 & 0.12 & 0.09 & 0.05 & 0.11 & 0.08 \\
\hline $\mathrm{Ga}$ & 8 & 7.5 & 4.9 & 12.5 & 5.6 & 5.9 & 12.5 & 10.7 & 11.5 & 9.1 & 10.2 & 10.9 \\
\hline $\mathrm{Gd}$ & 0.35 & 0.26 & 0.7 & 0.34 & 0.29 & 0.33 & 0.19 & 0.31 & 0.31 & 0.15 & 0.3 & 0.24 \\
\hline $\mathrm{Hf}$ & $<0.1$ & 0.2 & 0.1 & 0.1 & $<0.1$ & 0.1 & $<0.1$ & $<0.1$ & $<0.1$ & $<0.1$ & 0.2 & $<0.1$ \\
\hline Ho & 0.11 & 0.09 & 0.21 & 0.1 & 0.12 & 0.11 & 0.07 & 0.11 & 0.11 & 0.05 & 0.11 & 0.09 \\
\hline $\mathrm{La}$ & 0.3 & 0.3 & 0.2 & 0.5 & 0.2 & 0.2 & 0.2 & 0.3 & 0.3 & 0.2 & 0.2 & 0.2 \\
\hline $\mathrm{Lu}$ & 0.06 & 0.04 & 0.09 & 0.05 & 0.06 & 0.07 & 0.05 & 0.05 & 0.05 & 0.03 & 0.05 & 0.05 \\
\hline $\mathrm{Nb}$ & 0.1 & 0.2 & 0.1 & 0.1 & 0.1 & $<0.1$ & $<0.1$ & $<0.1$ & 0.1 & $<0.1$ & $<0.1$ & 0.1 \\
\hline $\mathrm{Nd}$ & 0.4 & 0 & 0.7 & 0.6 & 0.3 & 0.3 & 0.2 & 0.4 & 0.4 & 0.1 & 0.2 & 0.3 \\
\hline $\mathrm{Pr}$ & 0.07 & 0.06 & 0.09 & 0.12 & 0.04 & 0.04 & 0.03 & 0.07 & 0.07 & 0.02 & 0.04 & 0.05 \\
\hline $\mathrm{Rb}$ & 1.1 & 0.9 & 0.7 & 1.8 & 0.3 & 0.2 & 0.4 & 0.5 & 0.8 & 0.3 & 0.2 & 0.6 \\
\hline $\mathrm{Sm}$ & 0.19 & 0.13 & 0.34 & 0.19 & 0.13 & 0.16 & 0.1 & 0.16 & 0.16 & 0.06 & 0.15 & 0.12 \\
\hline $\mathrm{Sr}$ & 47 & 41.6 & 21.6 & 81.5 & 16.4 & 17.9 & 58.6 & 84.1 & 72.9 & 46.2 & 50.6 & 49.1 \\
\hline $\mathrm{Tb}$ & 0.08 & 0.05 & 0.14 & 0.06 & 0.06 & 0.07 & 0.05 & 0.06 & 0.06 & 0.03 & 0.06 & 0.04 \\
\hline Th & 0.08 & 0.05 & $<0.05$ & 0.14 & $<0.05$ & $<0.05$ & $<0.05$ & $<0.05$ & $<0.05$ & $<0.05$ & $<0.05$ & $<0.05$ \\
\hline $\mathrm{Tm}$ & 0.06 & 0.04 & 0.1 & 0.05 & 0.06 & 0.06 & 0.04 & 0.05 & 0.05 & 0.03 & 0.05 & 0.04 \\
\hline $\mathrm{U}$ & $<0.05$ & $<0.05$ & $<0.05$ & 0.07 & $<0.05$ & $<0.05$ & $<0.05$ & $<0.05$ & 0.17 & $<0.05$ & $<0.05$ & $<0.05$ \\
\hline V & 221 & 169 & 206 & 161 & 199 & 169 & 472 & 169 & 191 & 138 & 165 & 294 \\
\hline Y & 3 & 2.2 & 5.8 & 2.6 & 3 & 3 & 1.9 & 2.8 & 2.7 & 1.4 & 2.7 & 2.2 \\
\hline $\mathrm{Yb}$ & 0.38 & 0.25 & 0.64 & 0.3 & 0.4 & 0.41 & 0.25 & 0.3 & 0.34 & 0.18 & 0.34 & 0.27 \\
\hline $\mathrm{Zr}$ & 2 & 9 & $<2$ & 2 & $<2$ & 5 & $<2$ & $<2$ & $<2$ & $<2$ & 8 & $<2$ \\
\hline
\end{tabular}



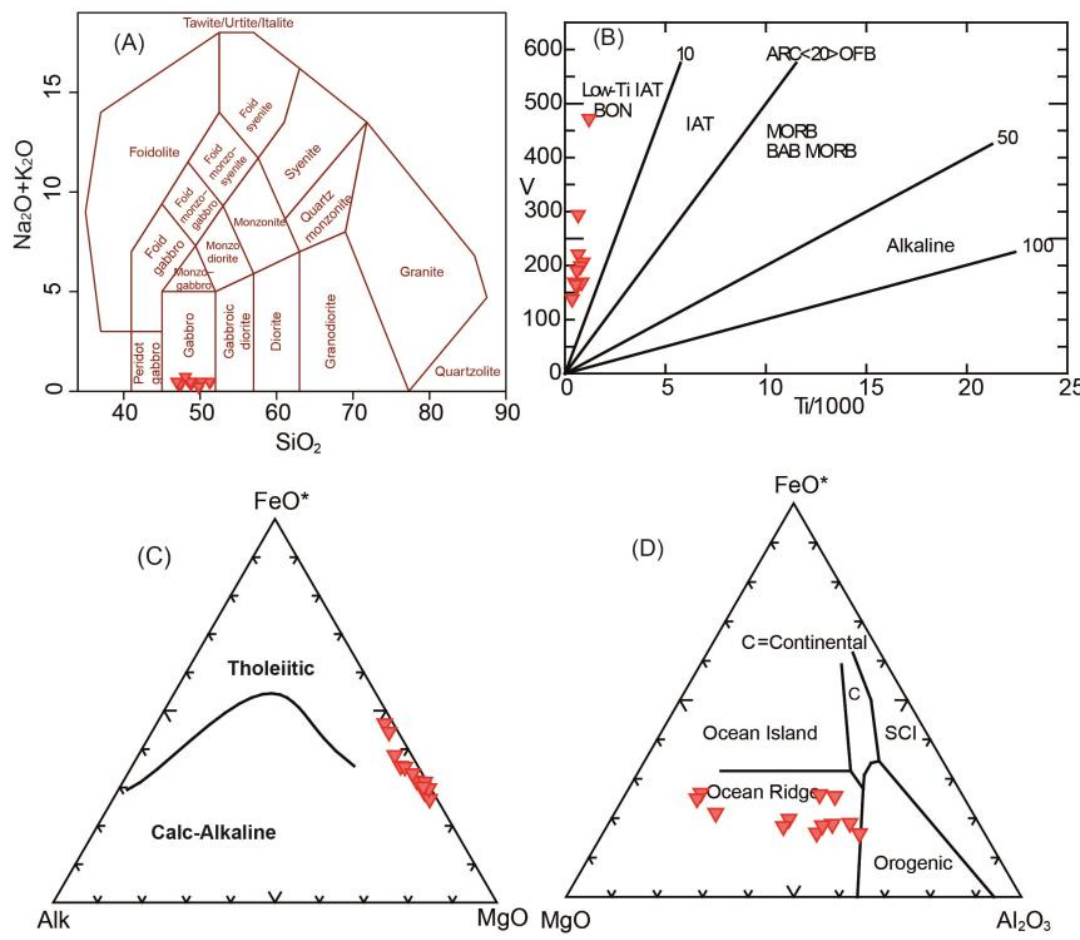

Fig.6. (A) Classification of the gabbroic rocks using the diagram of Middlemost 1985; (B) Tectonic discrimination diagram between $\mathrm{V}$ and Ti/1000 (after Shervais, 1982); (C) Alkaline $\left(\mathrm{Na}_{2} \mathrm{O}+\right.$ $\left.\mathrm{K}_{2} \mathrm{O}\right)-\mathrm{FeO}_{\text {total }}-\mathrm{MgO}(\mathrm{AFM})$ discrimination diagram for tholeiitic and calc-alkaline series after Irvine and Baragar (1971) (D) $\mathrm{MgO}-\mathrm{FeO}^{*}-\mathrm{Al}_{2} \mathrm{O}_{3}$ ternary discrimination diagram of Pearce and Gale, 1977

\subsection{Petrogensis of Penjwin Gabbroic Rocks}

Cr vs Y diagram indicates depleted mantle source show that amount of $\mathrm{Y}$ is nearly constant with variation in $\mathrm{Cr}$; this is a typical feature of depleted mantle source (Shinjo et al., 2000). In addition, Fig. 7 $\mathrm{A}$ and $\mathrm{B}$ shows a simple equilibrium partial melting diagram adapted to model primary melt composition from a simple mantle lherzolite having about 2000 ppm Cr (Pearce 1982; Malpas et al., $1994)$.The Penjwin gabbroic rocks, showing partial melting trends ( $30 \%$ to $\sim 60 \%)$ of upper depleted (Probably a lherzolitic) mantle source and fractional crystallization paths (A) for all lower layered gabbroic rocks and fractional crystallization paths (B) for all upper gabbroic rocks (see Fig.7). The Parallel-Subparallel and flat REE patterns of the Penjwin gabbroic rocks may indicate that they have originated from the same depleted mantle source which may dominated by harzburgite and dunite with local occurrence of lherzolite. All Penjwin gabbroic rocks show depleted in HFSEs in comparison with primitive mantle and display an arched upward REE pattern, characterized by LREE depletion $(\mathrm{LaN} / \mathrm{SmN}=0.05-0.8)$ and enrichment in MREE and HRE (Fig.7C and D). Moreover, the tholeiitic-transitional gabbro is REE- and HFS-depleted relative to primitive mantle, indicating derivation from melting of a refractory mantle peridotite source, possible spinel lherzolite (Bonev and Stampfli, 2009). All gabbroic samples have relatively high LILE/HFSE ratios and negative $\mathrm{Ta}-\mathrm{Nb}-\mathrm{Ti}$ and $\mathrm{Ce}$ anomalies, typical for subduction zone-related settings (Fig.7 $\mathrm{C}$ and D). Furthermore, the depletion in high field strength elements relative to fluid-mobile elements (negative and $\mathrm{Nb}$ anomalies comparing to the neighbouring incompatible elements) in all Penjwin gabbroic samples on a primitive-normalized plot (Fig. 7D), which are common in arc settings and are usually attributed to subduction enrichment and fluid metasomatism processes in subduction zones, Pearce and Peate, (1995) or it is explained by retention of these elements in the residual mantle source during partial melting 
(Pearce, 1982; Wilson, 1989). Negative Ti anomalies in the patterns for all studied samples are due to early crystallization of Fe-Ti oxides which buffers the Ti concentration or the former removal of $\mathrm{Fe}$ - $\mathrm{Ti}$ phases, ( due to the fractionation of ilmenite and titanomagnetite; cf Ozgenc and Ilbeyli (2009) which is consistent with the petrographic evidences (Fig. 4 F). The geochemical evidences show that Penjwin gabbroic rocks may be formed in the early stages of intraoceanic young supra-subduction zone (SSZ) at a Palaeo-ridge axis or close to it which led to contemporaneous eruptions in a fore-arc setting of island arc tholeiitic basalts (Fig.6B, C, D).
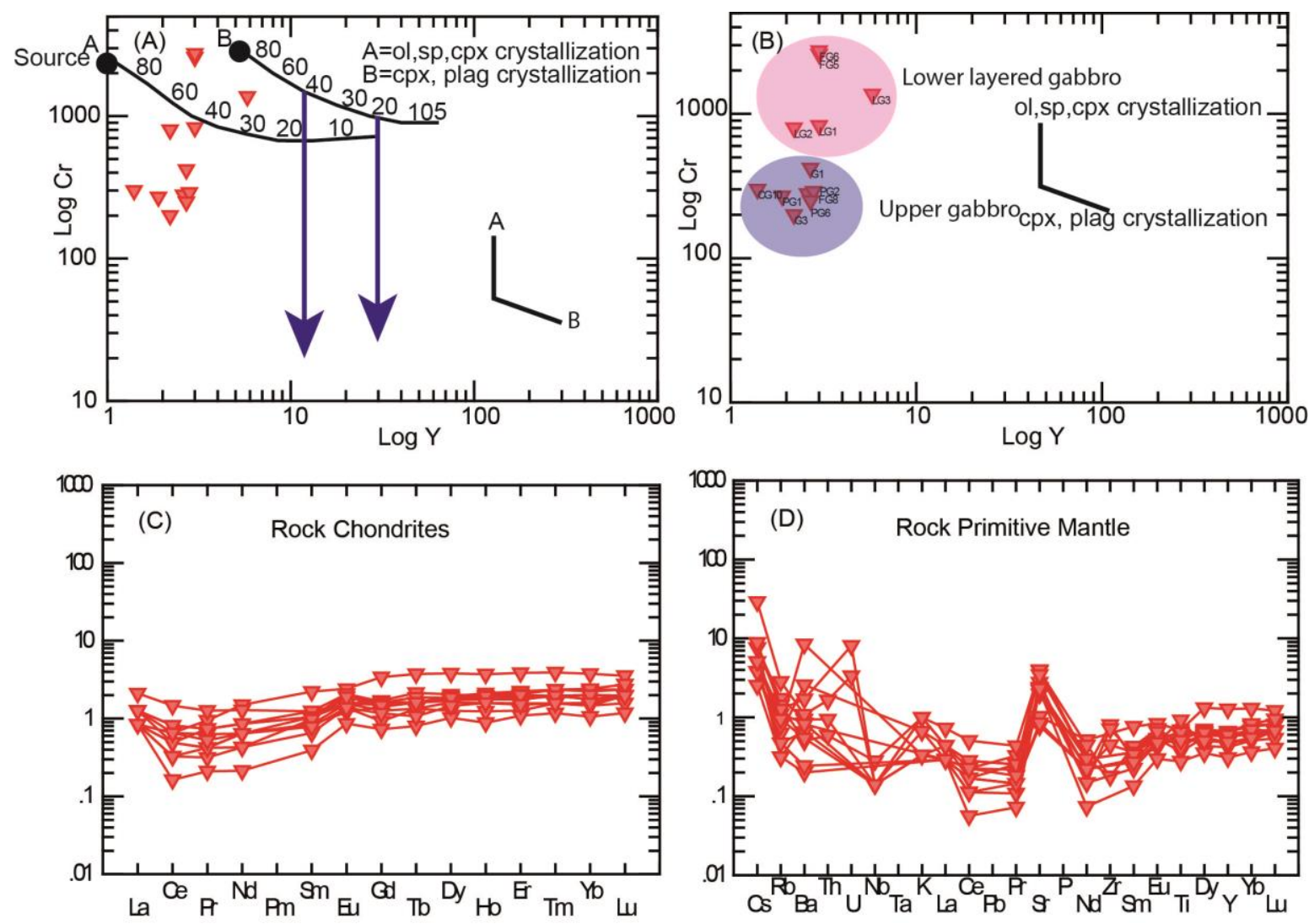

Fig.7. (A) Cr vs Y log plot for the Penjwin gabbroic rocks, showing partial melting trends of a lherzolitic mantle source and fractional crystallization paths for all the gabbros (modified after Malpas et al., 1994); (B) showing fractional crystallization paths for all the gabbros (C) chondrite normalized and (D) Primitive normalized rare-earth plot of Sun and McDonough (1989)

\section{Conclusions}

Our finding in this research show the gabbroic rocks in Penjwin ophiolite complex is a part of Zagros ophiolite and it would be more consistent with the other parts of ophiolite members such as Pillow lava, deeply basin sediments such as chert, and radiolarite, layered gabbro, sheeted dikes and mantle taconites with mainly harzburgite composition. These sequences have a clear correlation with the typical ophiolite members in the middle oceanic ridge and/or supra-subduction zone ophiolite. These rocks continued to the Iran side in Marivan (Rezaei et al., 2021) and Baneh (Azizi et al., 2019) area with 40-37Ma radiometric ages. Therefore, new detailed age dating of gabbroic rocks in the Penjwin area is needed to make their relation to the Zagros ophiolite and or syn-to post collision magmatism in the Zagros suture zone. 


\section{Acknowledgements}

This paper is a part of second author MSc thesis undertaken at the Department of Applied Geology, University of Kirkuk, and was supported financially by the University of Kirkuk. The authors are very grateful to the Editor in Chief Prof. Dr. Salih M. Awadh, the Secretary of Journal Mr. Samir R. Hijab. and the Technical Editors for their great efforts and valuable comments.

\section{References}

Abdulla, K. L., 2015. Petrogenesis and Geochronology of Plagiogranite Rocks in Penjween Ophiolite, Kurdistan Region, NE Iraq. M.Sc. thesis. College of Science, University of Sulaimani 88 pp.

Agard, P., Omrani, J., Jolivet, L., Mouthereau, F., 2005. Convergence history across Zagros (Iran): constraints from collisional and earlier deformation. International Journal Earth Science, 94, 401-419.

Alavi, M., 1994. Tectonic of the Zagros orogenic belt of Iran: new data and interpretations. Tectonophysics 229, 211-238.

Ali, S.A., Buckman, S., Aswad, K.J., Jones, B.G., Ismail, S., Nutman, A.P., 2012. Recognition of Late Cretaceous Hasanbag ophiolite arc rocks in the Kurdistan region of the Iraqi Zagros Thrust Zone: a missing link in the paleogeography of the closing Neo- Tethys Ocean. Lithosphere 4, 395-410.

Ali, S.A., Buckman, S., Aswad, K.J., Jones, B.G., Ismail, S., Nutman, A.P., 2013. The tectonic evolution of a Neo-Tethyan (Eocene-Oligocene) island arc (Walash-Naopurdan Group) in the Kurdistan region of the NE Iraqi Zagros Thrust Zone. Island Arc Journal 22, 104-125.

Ali, S.A., Ismail, S.A., Nutman, A.P., Bennett, V.C., Jones, B.G., Buckman, S., 2016. The intra-oceanic Cretaceous ( $\sim 108 \mathrm{Ma}$ ) Kata-Rash arc fragment in the Kurdistan segment of Iraqi Zagros suture zone: implications for Neotethys evolution and closure. Lithos 260, 154-163.

Ali, S.A., Mohajjel, M., Aswad, K.J., Ismail, S.A., Buckman, S., Jones, B.G., 2014. Tectono-stratigraphy and general structure of the northwestern Zagros collision zone across the Iraq-Iran border. Journal Environment Earth Science, 4 (4), 92e110.

Ali, S.A., Nutman, A.P., Aswad, K.J., Jones, B.G., 2019. Overview of the tectonic evolution of the Iraqi Zagros thrust zone: sixty million years of Neotethyan ocean subduction. Journal of Geodynamics, 129, 162-177.

Al-Hassan, M.E. and Hubbard, F, H., 1985. Magma segregation in the tectonic remnant of Basalt ophiolite Penjween NE Iraq. Ophiolite, 10, 139-146.

Al- Hassan, M. 1982. Petrology, mineralogy and geochemistry of the Penjwin Igneous complex, northeast Iraq. $\mathrm{Ph} . \mathrm{D}$. Thesis, University of Dande.

Aswad, K. J., 1999. Arc-continent collision in northeastern Iraq as evidenced by Mawat and Penjwin Ophiolite Complexes. Rafidain Journal of Sciences, 10, 51-61.

Aswad, K. J., Al-sheraefy, R.M., and Ali, S.A., 2013. Pre-collisional intrusive magmatism in the Bulfat Complex, Wadi Rashid, Qala Deza, NE Iraq: Geochemical and mineralogical constraints and implications for tectonic evolution of granitoidgabbro suites. Iraqi National Journal of Earth Science, 13, 103-137.

Aswad, K. J., Aziz, N.R. and Koyi, H.A., 2011. Cr-spinel compositions in serpentinites and their implications for the petrotectonic history of the Zagros Suture Zone, Kurdistan Region, Iraq. Geological Magazine, 148, 802-818.

Azizi, H. and Moineraziri, H., 2009. Review of the tectonic setting of Cretaceous to Quaternary volcanism in northeast Iran. Journal of Geodynamics, 47, 167-179.

Aziz, N.R, Sadiq D. M, and Aswad K. J., 2021. U-Pb detrital zircon dating of Middle Eocene clastic rocks from the Gercus Formation, NE Iraq: New constraints on their provenance, and tectonic evolution, Iraqi Geological Journal, 54 (1C),1-15.

Azizi, H., Hadad, S., Stern, R. J. \& Asahara, Y. 2019. Age, geochemistry, and emplacement of the 40 Ma Baneh granite-appinite complex in a transpressional tectonic regime, Zagros suture zone, northwest Iran. International Geology Review, 61, 195-223.

Bonev, N., and Stampfli, G., 2009, Gabbro, plagiogranite and associated dykes in the supra-subduction zone Evros Ophiolites, NE Greece: Geological Magazine,146, (1),72-91. 
Coogan, L. A., Saunders, A. D., Kempton, P. D., \& Norry, M. J. 2000. Evidence from oceanic gabbro for porous melt migration within a crystal mush beneath the Mid-Atlantic Ridge. Geochemistry, Geophysics, Geosystems, 1(9).

Furnes, H., Dilek, Y., Zhao, G., Safonova, I., \& Santosh, M. .2020. Geochemical characterization of ophiolites in the Alpine-Himalayan Orogenic Belt: Magmatically and tectonically diverse evolution of the Mesozoic Neotethyan oceanic crust. Earth-Science Reviews, 103258.

Irvine, T. H., Baragar, W. R. A. 1971. A guide to the chemical classification of the common volcanic rocks. Canadian Journal of Earth Sciences, 8, 523-48.

Jassim, S.Z., Goff, J.C., 2006. Geology of Iraq. Dolin, Prague, Moravian Museum, Brno, Czech Republic, 341 pp. Mahmmod, L.A., 1978. Petrology and geochemistry of ultramafics around Penjwin, northeast Iraq with special reference to the genesis of the chromites associated with them, M.Sc. thesis University of Mousl, Iraq, 220 pp.

Malpas, J., Calon, T., and Macdonald, R., 1994. The Shulaps Ophiolite Complex of British Columbia, Canada: a Palaeozoic, Mesozoic arc-related micro terrane. In Proceedings of the 29th International Geological Congress: Circum-Pacific, 1994. VSP Intl Science, 69-87.

Middlemost, E. A. K. 1985. Magmas and Magmatic Rocks: An Introduction to Igneous Petrology, London, United Kingdom, 266 pp.

Moghadam, H. S., and Stern, R.J., 2011. Late Cretaceous Fore-arc ophiolites of Iran (pictorial article). The Island Arc, 20, 1-4.

Moghadam, H. S., Corfu, F., Stern, R.J., Lotfibakhsh, A., 2019. The Eastern Khoy metamorphic complex of NW Iran: a Jurassic ophiolite or continuation of the Sanandaj- Sirjan Zone. Journal Geological Society. London, $176,517-529$.

Moghadam, H. S., Stern, R. J., 2015. Ophiolites of Iran: Keys to understanding the tectonic evolution of SW Asia: (II) Mesozoic ophiolites. Journal Asian Earth Science, 100, 31-59.

Mohammad, O., Ali, S. A., Aziz, N. R., Yara, I. O., Abdulla, K. L., 2021. Comment on "Generation and exhumation of granitoid intrusions in the Penjween ophiolite complex, NW Zagros of the Kurdistan region of Iraq: Implications for the geodynamic evolution of the Arabia- Eurasia collision zone, Journal of Lithosphere, 105915, 4.

Mohammad, Y.O., Cornell, D.H., 2017. U-Pb zircon geochronology of the Daraban leucogranite, Mawat ophiolite, Northeastern Iraq: a record of the subduction to collision history for the Arabia-Eurasia plates. Island Arc, 26, e12188.

Mohammad, Y.O., Cornell, D.H., Qaradaghi, J.H., Mohammad, F.O., 2014. Geochemistry and Ar-Ar muscovite ages of the Daraban Leucogranite, Mawat Ophiolite, northeastern Iraq: implications for Arabia-Eurasia continental collision. Journal Asian Earth Science, 86, 151-165.

Mohammad, Y. M., 2008. Origin of titanite in metarodingite from the Zagros Thrust Zone, Iraq. American Mineral., 93, 1133-1141.

Ozgenc, I., and Ilbeyli, N., 2009. Geochemical constraints on petrogenesis of Late Cretaceous alkaline magmatism in east -central Anatolia (Hasancelebi-Basoren, Malatya), Turkey. Mineralogy and Petrology, 95, 71-85.

Pearce, J.A., and Gale, G.H., 1977. Identification of ore deposition environment from trace element geochemistry of associated igneous host rocks, in volcanic processes in Ore Genesis, Gass, Geological Society London, 7, 14-24.

Pearce, J.A., and Peate, D.W., 1995, Tectonic implications of the composition of volcanic arc magmas. Annual Review of Earth and Planetary Sciences, 23, 251-285.

Pearce, J.A., 1982. Trace element characteristics of lavas from destructive plate boundaries, in Thorpe, R.S., (ed.), Orogenic Andesites: Chichester, Wiley, 528-548.

Rezaei, F., Azizi, H., \& Asahara, Y. 2021.Tectonic significance of the late Eocene (Bartonian) calc-alkaline granitoid body in the Marivan area, Zagros suture zone, northwest Iran. International Geology Review, $1-16$.

Schulz, B., Sandmann, D., Gilbricht, S., 2020. SEM-based automated mineralogy and its application in geo- and material sciences. Minerals 10, 1004.

Shervais, J.W., 1982. The petrochemistry of ophiolite gabbroic complexes: a key for the classification of ophiolites into low-Ti and high-Ti types. Earth Planet Science, 52, 203-212. 
Shinjo, R., Woodhead, J.D., Hergt, J.M., 2000. Geochemical variation within the northern Ryukyu Arc: magma source compositions and geodynamic implications, Contributions to Mineralogy and Petrology, 140, 263-282.

Stampfli, G.M., Borel, G.D., 2002. A plate tectonic model for the Paleozoic and Mesozoic constrained by dynamic plate boundaries and restored synthetic oceanic isochrones. Earth Planet Science, 196, 17-33.

Sun, S. S., and McDonough, W., 1989. Chemical and isotopic systematics of oceanic basalts: implications for mantle composition and processes. In: Saunders, A. D., London, Special Publication, 42, 313-345.

Talbot, C.J., Alavi, M., 1996. The past of a future syntaxis across the Zagross. Geological Society of London, 89-109.

Wilson, M.,1989. Igneous Petrogenesis: A Global Tectonic Approach. Unwin Hyman, London, 466 pp.

Znad, R. K., Mostafa, S. E., \& Al-Sumaidaie, M. A. (2020). Effect of tectonic setting on distribution of pelagic sediment unit (Shiranish Formation) in Zagross foreland basin, Iraqi segment. The Iraqi Geological Journal, 53 (2A), 105-119. 